\title{
Palmprint based Verification System Robust to Occlusion using Low-order Zernike Moments of Sub-images
}

Badrinath G. S.

badri@cse.iitk.ac.in

Naresh Kumar Kachhi

nareshb@iitk.ac.in

Phalguni Gupta

pg@iitk.ac.in
Dept. of Computer Science and Engg., Indian Institute of Technology Kanpur, Kanpur, India

Pin 208016

\begin{abstract}
This paper proposes a palmprint based verification system using low-order Zernike moments of palmprint sub-images. The Zernike moments of corresponding sub-images of live and enrolled palmprint are matched using Euclidean distance for verification. An efficient weighted fusion technique to fuse matching scores of the sub-images is proposed. The palmprint is extracted from the hand image acquired using a low cost flat bed scanner. The extraction is robust to hand translation and rotation on the scanner. The proposed system is also robust to occlusion and can verify user the features of from non-occluded region. The system is tested on IITK, PolyU and CASIA databases of size $549,5,239$ and 7,752 hand images respectively.
\end{abstract}

\section{Introduction}

Use of palmprint which is the region between wrist and fingers as biometric feature is relatively new a approach. Palmprint has features like texture, wrinkles, principle lines, ridges, and minutiae points that can be used for its representation $[\square]$. Palmprint has relatively stable and unique features. Data acquisition is easy and non-intrusive. Co-operation from the user to collect data is low. Devices to collect data are economic. It uses low resolution images and provides high efficiency. System based on palmprint has high user acceptably [ष]. Furthermore, palmprint also serves as a reliable biometric features because the print patterns are not same even in mono zygotic twins [0].

Limited work has been reported on palmprint based identification/verification despite of its significant features. Efforts have been made to build a palmprint based recognition system based on structural features of palmprint like crease points [ $[$ [], line features [0], Datum points [ $\square]$ ], local binary pattern histograms [ $\square$ ]. There also exists systems based on statistical features of palmprint extracted using Fourier transforms [四], Discrete Cosine Transforms [0], Karhunen-Lowe transforms [四], Wavelet transforms [ $\mathbf{Q}, \mathbf{\square}]$ ], Independent Component Analysis, Gabor filter [ㅁ] ], Linear Discriminant Analysis(LDA) []], Neural networks $[\boldsymbol{G}, \mathbf{\Xi}]$, statistical signature $[\mathbb{\square}]$ and hand geometry $[\boldsymbol{\Xi}]$. Furthermore there are 
multi-modal biometric systems fusing feature from other traits like fingerprint [四], hand geometry [छ], and face [ $\square]$ to improve the accuracy.

The palmprint based systems in $[\square, \square]$ for verification uses ink marking to capture the palmprint patterns. These systems are not widely accepted because of high attention and cooperation of users to provide data. In recent papers based on palmprint images are captures using digital camera [ $[\mathbf{\square}]$ and users hand is placed in constrained environment using pegs. The problem with this system are user may fail to provide the sample if he is injured or physically challenged. Hence there is a need to build a system which is

- Constraint free image acquisition: Device to acquire hand image should be constraint free so that physically challenged or injured people can provide biometric sample.

- Robust to translation of hand image on the scanner: System must be able to extract palmprint independent of translation, rotation or placement of hand on scanner surface.

- Robust to occlusion of hand image: If user exposes partial palmprint due to injuries or physically challenged, system should be able to verify.

- High Accuracy: High Accuracy with reasonable price to suit high end security applications.

This paper proposes a palmprint based biometric system that addresses some of the above characteristics. The features are extracted from small partitions (sub-images) of the palmprint using Zernike moments. The matching scores of sub-images are fused using weighted sum rule for decision. The hand images for feature extraction are acquired using low cost flat bed scanner.

The rest of the paper is organized as follows; Section 2 describes the Zernike moments which have been used in proposed system to extract features of the palmprint. Next section proposes system based on weighted and non weighted sum of sub-images matching scores. The experimental results on different datasets and robustness of the system to occlusion are reported in Section 4. Conclusion is presented in last section.

\section{Zernike Moments}

Zernike moments are generated using zernike polynomial which are orthogonal. Orthogonal features have the property of low redundancy, so every moment has unique information about the image. Higher the number of moments greater the details of image. Zernike moments are also invariant to rotation and translation, and relatively less sensitive to noise. Thus extracted zernike moments from an image can be matched correctly with high probability against features from large database.

Zernike moments can be defined as a set of orthogonal Zernike polynomial defined inside a unit circle. The Zernike moments of order $p$ and repetition $q$ for an image $I(r, \theta)$, over the polar coordinate space are obtained [ $\mathbb{}$ ] as

$$
Z_{p q}=\frac{p+1}{\Pi} \int_{0}^{2 \Pi} \int_{0}^{1} R_{p q}(r) \cdot e^{\hat{i} q \theta} I(r, \theta) r d r d \theta, \hat{i}=\sqrt{-1}
$$




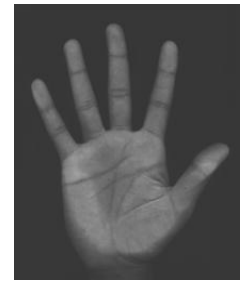

(a)

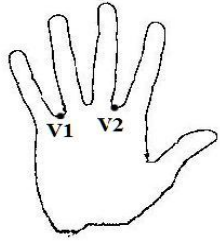

(b)

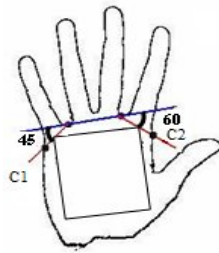

(c)

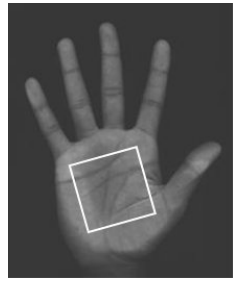

(d)

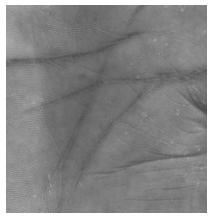

(e)

Figure 1: (a) Scanned Image. (b) Hand Contour and reference points. (c) Relevant points and Region of interest (palmprint). (d) Region of interest in gray scale hand image (e) Extracted Region of interest (palmprint)

where $R_{p q}$ is a real valued radial polynomial defined as-

$$
R_{p q}(r)=\sum_{n=0}^{(p-|q|) / 2}(-1)^{n} \frac{(p-n) !}{n !\left(\frac{p+|q|}{2}-n\right) !\left(\frac{p-|q|}{2}-n\right) !} r^{p-2 n}
$$

where $0 \leq|q| \leq p$ and $p-|q|$ is even. For an input image $I$ of size $N \times N$, equation for Zernike moment can be approximated as

$$
Z_{p q}=\lambda(p, N) \sum_{i=0}^{N-1} \sum_{j=0}^{N-1} R_{p q}\left(r_{i j}\right) e^{-\hat{i} q \theta_{i j}} I(i, j)
$$

where $r_{i j}=\sqrt{x_{i}^{2}+y_{j}^{2}}, \theta_{i j}=\tan ^{-1}\left(\frac{y_{j}}{x_{i}}\right), x_{i}=c_{1} i+c_{2}$ and $y_{j}=c_{1} j+c_{2} \cdot c_{1}, c_{2}$ and $\lambda(p, N)$ are normalized constants.

\section{Proposed System}

The proposed palmprint based verification system which robust to occlusion starts with acquiring the input image of hand using low cost scanner. The acquired hand image is preprocessed and region of interest is extracted. In the next step the extracted palmprint is enhanced to improve the texture. The palmprint is partitioned into sub-images and its features are extracted using Zernike moments in feature extraction module. IN the next step features of live palmprint sub-image are matched with corresponding sub-images of enrolled palmprint in the database. The matching scores of all sub-images are fused using weighted sum rule. Matching decision is done based on threshold.

\subsection{Image Acquisition}

Hand image from users are obtained in gray scale at spatial resolution of 200 dots per inch using a flat-bed scanner. Typical gray level image obtained from the scanner is shown in 1(a). The device used to obtain hand image is constraints (pegs) free, so user is free to place hand independent to rotation relative to line of symmetry of the working surface of scanner. 


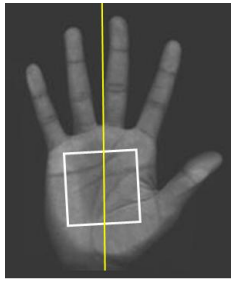

(a)

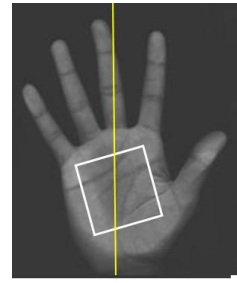

(b)

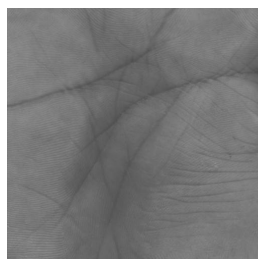

(c)

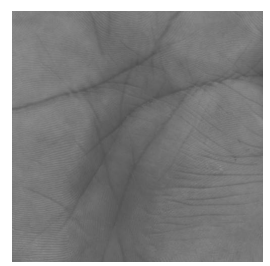

(d)

Figure 2: (a) and (b) Hand images of same user with different orientation and placement relative to symmetry of scanner surface. (c) and (d) Extracted palmprint for hand images shown in 2(a) and 2(b) respectively.

\subsection{Pre-processing and Region of Interest Extraction}

In this section the hand image is pre-processed and palmprint region is extracted. The acquired hand image is binarised using global thresholding. The contour of the binarised images is computed using [ $[\mathrm{\theta}]$. Two valley points $(V 1, V 2)$ between fingers are detected on the contour of the hand image as shown in Fig. 1(b).

Square area as shown in Fig 1(c) with two of its adjacent points coinciding the mid-points of line segments $V 1-C 1$ and $V 2-C 2$ is considered as region of interest or palmprint. The line segments $V 1-C 1$ and $V 2-C 2$ are inclined at an angle of $45^{\circ}$ and $60^{\circ}$ to line joining $V 1-V 2$ respectively. The region of interest in gray scale hand image and extracted region of interest in gray scale is shown in Fig. 1(d) and Fig. 1(e) respectively.

Since the hand image acquisition device is pegs free, orientation of placement of palm on the scanner surface would vary for every incident. Hand images with different orientation of placement for same user are shown in Fig. 2(a) and Fig. 2(b). The extracted region of interest is relative to the valley points and which are stable for the user. So the extracted palmprint region with different orientation of placement for the same user remains unchanged as shown in Fig. 2(c) and 2(d). Hence the proposed palmprint extraction procedure of the system makes the system robust to rotation. The experimental results show that the system is robust to rotation for about $\pm 35^{\circ}$.

\subsection{Palmprint Enhancement}

Because of non-uniform reflection from the relatively curvature surface of the palm, the extracted palmprint does not have uniform brightness. To obtain the well distributed texture image following operations are applied on extracted palmprint.

(a) Palmprint is divided into $32 \times 32$ sub-blocks. Mean for each sub-block is calculated to estimate the reflection of the image.

(b) Estimated coarse reflection is expanded to original palmprint size using bi-cubic interpolation. Fig. 3(b) shows the estimated coarse reflection of Fig.3(a).

(c) Uniform brightness image is obtained by subtracting estimated reflection from the original image. Uniform brightness images of Fig. 3(a) is shown in Fig. 3(c).

(d) Histogram equalization is done on blocks of $64 \times 64$ to obtain the enhanced image. The enhanced image is shown in Fig. 3(d). 


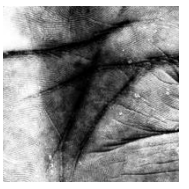

(a)

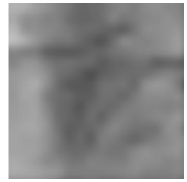

(b)

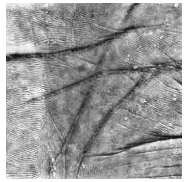

(c)

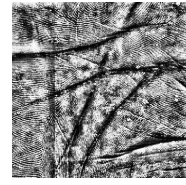

(d)

Figure 3: (a) Extracted palmprint. (b) Estimated coarse reflection. (c) Uniform brightness palmprint image (d) Enhanced palmprint image.

\subsection{Palmprint Sub-Image Feature Extraction}

The extracted and enhanced palmprint image is divided into $m \times m$ equal sized sub-images. Zernike moments extracted from the sub-images of the palmprint are used as features, which provides good discrimination ability and are used for palmprint based verification. The order of Zernike moments determine the detail of information regarding palmprint. Higher the order of moments, greater the details of the palmprint image. The Zernike moments of low-order are extracted from sub-images of palmprint are used as features. It is observed that palmprint features using low order Zernike moments of all sub-images is more discriminating than high order zernike moments of the entire palmprint (i.e $1 \times 1$ sub-image).

\subsection{Matching}

To verify the live palmprint, the Zernike moments computed for the sub-image of enrolled palmprint should be matched with Zernike moments of the corresponding sub-images of live palmprint. In this section fusion of matching scores of Zernike moments from all sub-images using non-weighted and weighted sum rule are presented.

(a) Non-weighted fusion of sub-image scores: To verify the live palmprint the Zernike moments of sub-images are matched with corresponding sub-image Zernike moments stored in database using Euclidean distance. The resulting matching scores of all subimages are fused using sum rule.

Let $L$ and $E$ be matrix of Zernike moments of live palmprint $(L I)$ and enrolled palmprint $(E I$ images partitioned into $(m \times m)$ sub-images respectively as:

$$
L=\left(\begin{array}{c}
l_{1,1} l_{1,2} \cdots l_{1, m} \\
l_{2,1} l_{2,2} \cdots l_{2, m} \\
\vdots \\
l_{m, 1} l_{m, 2} \cdots l_{m, m}
\end{array}\right) \quad E=\left(\begin{array}{c}
e_{1,1} e_{1,2} \cdots e_{1, m} \\
e_{2,1} e_{2,2} \cdots e_{2, m} \\
\vdots \\
e_{m, 1} e_{m, 2} \cdots e_{m, m}
\end{array}\right)
$$

The live palmprint $L I$ is assumed to be matched with enrolled palmprint $E I$ if

$$
\left(\sum_{i=1}^{m} \sum_{j=1}^{m}\left\|l_{i, j}-e_{i, j}\right\|\right) /(m \times m)<T
$$

where $\left\|l_{i, j}-e_{i, j}\right\|$ is the Euclidean distance between the Zernike moments $l_{i, j}$ of subimage $(i, j)$ in $L$ and Zernike moments $e_{i, j}$ of sub-image $(i, j)$ in $E$.

(b) Weighted fusion of sub-image scores: It can be observed that the certain sub-images are rich in texture and principle lines relative to other sub-images. So these sub-images 
have more discriminating ability compared to other sub-images. Hence matching scores of sub-images are weighted based on its discrimination level to improve the performance of proposed system. The discrimination level $\operatorname{Disc}_{i, j}$ of sub-image $(i, j)$ is computed using

$$
\operatorname{Disc}_{i, j}=\left\|\operatorname{Sim}_{i, j}-\operatorname{Dis}_{i, j}\right\| / \max \left(\left\|\operatorname{Sim}_{i, j}-\operatorname{Dis}_{i, j}\right\|\right) \text {; }
$$

where $\left\|\operatorname{Sim}_{i, j}-D_{i s_{i, j}}\right\|$ is the Euclidean distance between the average Zernike moments similarity distance of sub-image $(i, j)$ for entire enrolled database, and Dis $s_{i, j}$ is Zernike moment average dissimilarity distance of sub-image $(i, j)$ for entire enrolled database. The average similarity $\operatorname{Sim}_{i, j}$ distance of the sub-image $(i, j)$ for entire database is computed using

$$
\operatorname{Sim}_{i, j}=\left(\sum_{p=1}^{N u m}\left(\sum_{r=1}^{k} \sum_{s=r+1}^{k}\left\|I_{i, j}^{p, r}-I_{i, j}^{p, s}\right\|\right)\right) /\left(N u m \times\left(\begin{array}{l}
k \\
2
\end{array}\right)\right)
$$

where $I_{i, j}^{p, r}$ is the Zernike moment of $(i, j)$ sub-image of $p^{t h}$ user and $r \in[1, k]$ enrolled images of $p^{\text {th }}$ user, Num is total number of registered users in database and $k$ is number of enrolled images per user.

The average dis-similarity distance of the sub-image $(i, j)$ for entire database is computed using

$$
D i s_{i, j}=\left(\sum_{p=1}^{N u m} \sum_{q=p+1}^{N u m}\left(\sum_{r=1}^{k} \sum_{s=1}^{k}\left\|I_{i, j}^{p, r}-I_{i, j}^{q, s}\right\|\right)\right) /\left(N u m \times \frac{(N u m-1) k}{2} \times k\right)
$$

Matching distances of sub-images using weighted sum rule with discrimination levelDisc $c_{i, j}$, the live palmprint image $L I$ is matched with Enrolled palmprint image $E I$ if

$$
\left(\sum_{i=1}^{m} \sum_{j=1}^{m}\left\|l_{i, j}-e_{i, j}\right\| \times \operatorname{Disc}_{i, j}\right) /(m \times m)<W T h
$$

where $W T h$ is weighted threshold.

\section{Experimental Results}

The proposed system is tested on three sets of image databases available in Indian Institute of Technology Kanpur (IITK), Chinese Academy of Sciences Institute of Automation (CASIA)[] and Hong Kong Polytechnic University (PolyU)[ㅁ]].

\subsection{Datasets}

\subsubsection{Indian Institute of Technology Kanpur}

IITK has collected a 549 hand images from 150 subjects corresponding to 183 different palms. Three hand images per subject collected with the help of flat bed scanner at 200 dots per inch spatial resolution and 256 gray levels. The scanner is pegs free, so subject is independent to rotate his hand $\pm 35^{\circ}$ symmetric to working surface of scanner. One image per palm is considered for training and remaining two images are used for the testing. 


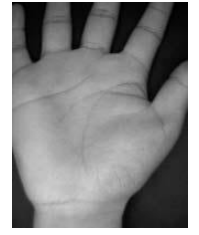

(a)

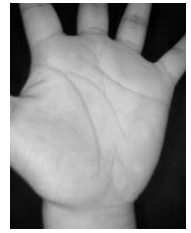

(b)

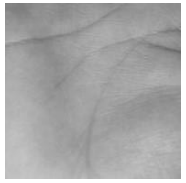

(c)

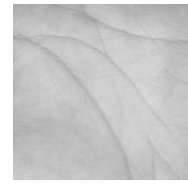

(d)

Figure 4: (a) and (b) are sample hand images from CASIA database. (c) and (d) are extracted palmprint from hand images shown in Fig. 4(a) and 4(b) respectively.

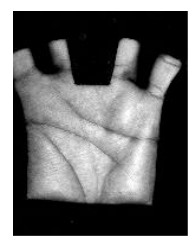

(a)

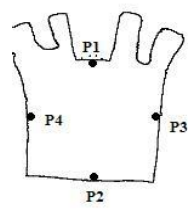

(b)

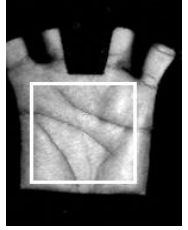

(c)

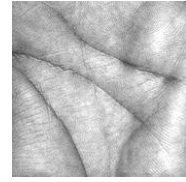

(d)

Figure 5: (a) PolyU sample image. (b) Reference points. (c) Region of interest in gray scale hand image (d) Extracted region of interset.

\subsubsection{CASIA Database}

CASIA database contains 5,239 hand images captured from 301 subjects corresponding to 602 palms. For each subject, around 8 images from left hand and 8 images from right hand are collected. All images collected using CMOS at spatial resolution of 96 dots per inch, and 256 gray-level. The device is pegs free, so the user is free to place his hand facing the scanner. Sample of hand images are shown in Fig. 4(a) and 4(b). Extracted palmprint for image 4(a) and 4(b) are shown Fig 4(c) and Fig 4(d) shows. Two images per palm is considered for training and remaining six images are used for the testing.

\subsubsection{PolyU Database}

Database from PolyU [四] of 7752 grayscale images from 193 subjects corresponding to 386 different palms. Around 17 images per palm have been collected in two sessions using CCD [ए]] at spatial resolution of 75 dots per inch, and 256 gray levels. Images are captured placing pegs. Fig. 5(a) shows the sample of database. Six images per palm is considered for training and remaining ten images are used for the testing.

Following method is proposed to extract the region of interest for PolyU database. Four reference points $P 1, P 2, P 3$ and $P 4$ are located on the contour of palm as shown in Fig. 5 (b). Square area of $200 \times 200$ pixels with its center coinciding with intersection of line segments $P 1-P 2$ and $P 3-P 4$ is considered as region of interest. Fig. 5(c) shows the region of interest in gray scale hand image, and the extracted palmprint image is shown in Fig. 5(d). 


\begin{tabular}{|l||c|l||c|l||l|l||l|l|}
\hline \multicolumn{1}{|c||}{} & \multicolumn{2}{c||}{ Order 1 } & \multicolumn{2}{c||}{ Order 3 } & \multicolumn{2}{c||}{ Order 6 } & \multicolumn{2}{c|}{ Order 8 } \\
\cline { 2 - 9 } & \multicolumn{2}{|c||}{ Sub-images } & \multicolumn{2}{c|}{ Sub-images } & \multicolumn{2}{c|}{ Sub-images } & \multicolumn{2}{c|}{ Sub-images } \\
\cline { 2 - 9 } & $1 \times 1$ & $10 \times 10$ & $1 \times 1$ & $10 \times 10$ & $1 \times 1$ & $10 \times 10$ & $1 \times 1$ & $10 \times 10$ \\
\hline ACC(\%) & 80.57 & 88.65 & 90.10 & 87.1 & 92.02 & 84.99 & 92.62 & 84.08 \\
\hline FAR(\%) & 25.03 & 4.27 & 9.92 & 6.06 & 3.45 & 4.35 & 2.25 & 3.55 \\
\hline FRR(\%) & 13.81 & 18.42 & 9.86 & 19.73 & 12.5 & 25.65 & 12.5 & 28.28 \\
\hline ERR(\%) & 20.40 & 13.15 & 9.92 & 16.56 & 3.79 & 20.40 & 9.86 & 19.14 \\
\hline
\end{tabular}

Table 1: Accuracy(ACC), FAR, FRR and ERR of the proposed system for IITK database.

\subsection{Results}

The experiments are conducted for moment orders ranging from 1 to 8 , and portioning the palmprint into $(2 \times 2),(3 \times 3),(4 \times 4),(5 \times 5),(6 \times 6),(7 \times 7),(8 \times 8),(9 \times 9)$ and $(10 \times$ 10) sub-images for all three datasets. Accuracy(ACC), False Acceptance Rate (FAR), False Rejection Rate (FRR) and Equal Error Rate (ERR) of the system is shown in Table 1, Table 2 and Table 3 for IITK, CASIA and PolyU databases respectively. From results it can be said that the proposed system with low order (1, and 3) Zernike moments as features from subimages $(10 \times 10)$ outperforms conventional method of high order Zernike moments features with high order $(6$ and 8$)$ for the whole palmprint (i.e. $1 \times 1$ sub-image). It is also observed that when image is divided into $(10 \times 10)$ sub-images, the performance of system for Zernike moments with order 1 is more than Zernike moments with higher order (3, 6 and 8).

The proposed system has fused the matching scores of sub-images using weighted sum rule with discrimination level Disc as weights for IITK, CASIA and PolyU databases. Results are compared with the best known system [ㅁ] in the literatures which extracts palmprint features using Gabor filterand theese for weighted (Wgt) and non-weighted (N-Wgt) fusion of matching scores, varying number of sub-image partitioning and order of the Zernike moments are given in Table 4. It can be observed that weighted fusion stratery is better than the non weighted. Also, The proposed system with non weighted or weighted fusion of matching scores of sub-images $(10 \times 10)$ performs better than the best known system [ष] in literature.

\subsection{Robust to occlusion}

The proposed system uses Zernike moments from the non-occluded sub-images as features for its representation. It can be noted that the extracted Zernike moments of a sub-image is independent to those of other sub-images. Hence the system is robust to occlusion. Since palmprint is rich in texture, the randomness of palmprint or sub-image is also high, if subimage is occluded the texture is low and the randomness is also low. Hence based on the randomness, the sub-image can be classified into occluded or non occluded. Entropy is used

\begin{tabular}{|l||l|l||l|l||l|l||l|l|}
\hline \multicolumn{1}{|c||}{} & \multicolumn{2}{c||}{ Order 1 } & \multicolumn{2}{c||}{ Order 3 } & \multicolumn{2}{c||}{ Order 6 } & \multicolumn{2}{c|}{ Order 8 } \\
\cline { 2 - 9 } & \multicolumn{2}{|c||}{ Sub-images } & \multicolumn{2}{c|}{ Sub-images } & \multicolumn{2}{c|}{ Sub-images } & \multicolumn{2}{c|}{ Sub-images } \\
\cline { 2 - 9 } & $1 \times 1$ & $10 \times 10$ & $1 \times 1$ & $10 \times 10$ & $1 \times 1$ & $10 \times 10$ & $1 \times 1$ & $10 \times 10$ \\
\hline ACC(\%) & 85.06 & 98.66 & 94.56 & 98.21 & 96.72 & 97.88 & 97.23 & 97.67 \\
\hline FAR(\%) & 18.50 & 0.74 & 4.42 & 0.31 & 2.87 & 0.85 & 1.96 & 0.92 \\
\hline FRR(\%) & 11.36 & 1.93 & 6.43 & 3.24 & 3.68 & 3.37 & 3.56 & 3.68 \\
\hline ERR(\%) & 15.11 & 1.81 & 5.73 & 2.37 & 3.43 & 2.56 & 2.87 & 2.90 \\
\hline
\end{tabular}

Table 2: Accuracy(ACC), FAR, FRR and ERR of the proposed system for CASIA database. 


\begin{tabular}{|l||c|l||l|l||l|l|||l|l|}
\hline \multicolumn{1}{|c||}{} & \multicolumn{2}{c||}{ Order 1 } & \multicolumn{2}{c||}{ Order 3 } & \multicolumn{2}{c||}{ Order 6 } & \multicolumn{2}{c|}{ Order 8 } \\
\cline { 2 - 9 } & \multicolumn{2}{|c||}{ Sub-images } & \multicolumn{2}{c|}{ Sub-images } & \multicolumn{2}{c|}{ Sub-images } & \multicolumn{2}{c|}{ Sub-images } \\
\cline { 2 - 8 } & $1 \times 1$ & $10 \times 10$ & $1 \times 1$ & $10 \times 10$ & $1 \times 1$ & $10 \times 10$ & $1 \times 1$ & $10 \times 10$ \\
\hline ACC $(\%)$ & 79.28 & 99.29 & 89.36 & 99.50 & 93.81 & 99.39 & 95.36 & 99.39 \\
\hline FAR(\%) & 24.78 & 0.24 & 7.04 & 0.07 & 3.14 & 0.04 & 2.19 & 0.04 \\
\hline FRR(\%) & 16.63 & 1.16 & 14.22 & 0.91 & 9.23 & 1.16 & 7.07 & 1.16 \\
\hline ERR(\%) & 21.56 & 1.0 & 11.40 & 0.71 & 6.57 & 0.83 & 4.74 & 0.91 \\
\hline
\end{tabular}

Table 3: Accuracy(ACC), FAR, FRR and ERR of the proposed system for PolyU database.

\begin{tabular}{|c|c|c|c|c|c|c|c|c|c|}
\hline & \multicolumn{3}{|c|}{ IITK } & \multicolumn{3}{|c|}{ CASIA } & \multicolumn{3}{|c|}{ PolyU } \\
\hline & \multicolumn{2}{|c|}{ Proposed } & & \multicolumn{2}{|c|}{ Proposed } & \multirow[b]{2}{*}{ Gabor[四] } & \multicolumn{2}{|c|}{ Proposed } & \\
\hline & N-Wgt & Wgt & Gabor [四] & N-Wgt & Wgt & & N-Wgt & $\overline{\text { Wgt }}$ & Gabor[ם] \\
\hline $\mathrm{ACC}(\%)$ & 88.65 & 98.74 & 85.90 & 98.66 & 98.74 & 96.44 & 99.29 & 99.31 & 98.56 \\
\hline FAR(\%) & 4.27 & 1.81 & 11.08 & 0.74 & 0.58 & 2.30 & 0.24 & 0.28 & 0.44 \\
\hline FRR(\%) & 18.42 & 1.93 & 17.10 & 1.93 & 1.93 & 4.80 & 1.16 & 1.08 & 0.83 \\
\hline $\operatorname{ERR}(\%)$ & 13.15 & 1.81 & 15.13 & 1.81 & 1.81 & 10.0 & 1.0 & 0.91 & 3.76 \\
\hline
\end{tabular}

Table 4: Accuracy (ACC), FAR, FRR and EER of the proposed system and [ष]

to measure the randomness of the sub-image and classify the sub-image as occluded or nonoccluded region. The sub-image $L I_{i, j}$ of live palmprint $L I$ is non-occluded if

$$
-\sum_{x=0}^{255}\left(L I_{i, j}^{x} * \log _{2}\left(L I_{i, j}^{x}\right)\right) \leq O c c T h
$$

where $L I_{i, j}^{x}$ is the number of pixels with intensity $x$ in $L I_{i, j}$ and $O c c T h$ is the threshold for randomness.

Using Eq. 10, the Eq. which becomes robust to occlusion for verification can be written as

$$
\left(\sum_{i=1}^{m} \sum_{j=1}^{m}\left\|l_{i, j}-e_{i, j}\right\| \times \operatorname{Disc}_{i, j}\right) \times O c c_{i, j} / \sum_{1 \leq i, j \leq m} O c c<W T h
$$

where $O c c_{i, j}$ is the binary map of live image $L I_{i, j}$ as

$$
O c c_{i, j}=\left\{\begin{array}{cc}
1 & \text { if }-\sum_{x=0}^{255}\left(L I_{i, j}^{x} * \log _{2}\left(L I_{i, j}^{x}\right)\right) \leq O c c T h \\
0 & \text { otherwise }
\end{array}\right.
$$

If the number of partitions of palmprint is low, large region of palmprint would be omitted for small occlusion. So to avoid omitting non-occluded region from matching, the palmprint is partitioned in to many sub-images. In this paper the palmprint is divided into $(10 \times 10)$ sub-images. Experimentally it is observed that for $(10 \times 10)$ partitions the Zernike features

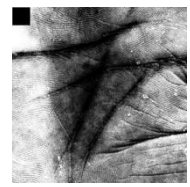

(a)

$0.1 W \times 0.1 H$

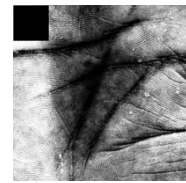

(b)

$0.2 \mathrm{~W} \times 0.2 \mathrm{H}$

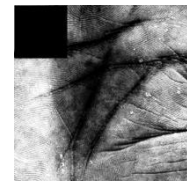

(c)

$0.3 W \times 0.3 H$

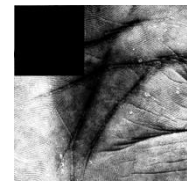

(d)

$0.4 W \times 0.4 H$

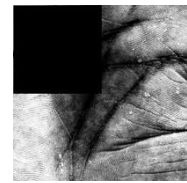

(e)

$0.5 W \times 0.5 H$

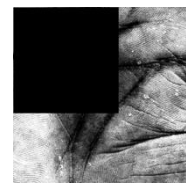

(f) $0.6 \mathrm{~W} \times 0.6 \mathrm{H}$

Figure 6: Typical occluded sizes used for testing the system. 


\begin{tabular}{|l|l|l|l|l|l|l|}
\hline Database & $0.1 W \times 0.1 H$ & $0.2 W \times 0.2 H$ & $0.3 W \times 0.3 H$ & $0.4 W \times 0.4 H$ & $0.5 W \times 0.5 H$ & $0.6 W \times 0.6 H$ \\
\hline IITK & $88.48 \%$ & $88.04 \%$ & $87.87 \%$ & $87.14 \%$ & $85.81 \%$ & $84.0 \%$ \\
\hline CASIA & $98.50 \%$ & $98.34 \%$ & $98.17 \%$ & $97.97 \%$ & $97.12 \%$ & $95.91 \%$ \\
\hline PolyU & $99.40 \%$ & $99.18 \%$ & $98.31 \%$ & $96.81 \%$ & $93.53 \%$ & $82.63 \%$ \\
\hline
\end{tabular}

Table 5: Accuracy for different sizes of occlusions

with lower order (i.e order one ) is performing better than higher order (i.e. 3, 6 and 8) moments on IITK , CASIA and PolyU. The testing set palmprint images are occluded by $0.1 W \times 0.1 H, 0.2 W \times 0.2 H, 0.3 W \times 0.3 H, 0.4 W \times 0.4 H, 0.5 W \times 0.5 H$ and $0.6 W \times 0.6 H$ size is considered to test the system where The $W$ and $H$ refer to width and height of the palmprint image. The typical occluded images used for testing are shown in Fig. 6.

The verification Accuracy, FAR, FRR and EER of the proposed system at different sizes of occlusion are shown in Table 5. From Table 5 it can be said that the claim of robustness of the proposed system is robust to justified.

\section{Conclusions}

This paper has proposed a palmprint verification system using low order zernike features from sub-images of palmprint. The systems performs better than conventional system which extracts high order Zernike moments from entire palmprint. A technique to extract palmprint region from the hand image obtained from flat bed scanner is also proposed. It is found to be robust to translation and rotation symmetric to scanner surface and can classify the sub-images as occluded or non-occluded based on the randomness the sub-image, which is entropy of the sub-image texture. Using only non-occluded sub-images the systems verifies the user, hence the proposed system is also robust to occlusion. The system performs with accuracy of $98.74 \%, 98.75 \%$ and $99.31 \%$ for IITK , CASIA and PolyU databases respectively. It has EER rate less than $2 \%$ for all three databases and performs better than the best known system [四] in literature for IITK, PolyU and CASIA databases. Thus the design of the system with robustness to occlusion, and robust to rotation and translation on scanner bed, and use of low cost scanner for acquisition of palm image has demonstrated the possibility of using this system for civilian and high end security applications.

\section{References}

[1] The polyu palmprint database. http://www.comp.polyu.edu.hk/ biometrics.

[2] The casia palmprint database. http://www.cbsria.ac.cn/.

[3] G.S. Badrinath and P. Gupta. An efficeint multi-algorithm fusion system based on palmpring for personnel identification. In Intl. Conf. on Advanced Computing, pages 759-764, 2007.

[4] J. Chen, C. Zhang, and G. Rong. Palmprint recognition using crease. In International Conference on Information Processing, pages 234-237, 2001.

[5] T. Connie, A.B.J. Teoh, M.G.K. Ong, and D.C.L. Ngo. An automated palmprint recognition system. Image and Vision Computing, 23(5):501-515, May 2005. 
[6] C. Han, H. Cheng, C. Lin, and K. Fan. Personal authentication using palm-print features. Pattern Recognition, 36(2):371-381, February 2003.

[7] X.Y. Jing and D. Zhang. A face and palmprint recognition approach based on discriminant det feature extraction. Systems, Man, and Cybernetics-B, 34(6):2405-2415, December 2004.

[8] A. Kumar and D. Zhang. Personal recognition using hand shape and texture. IEEE Transactions on Image Processing, 15(8):2454-2461, August 2006.

[9] T. Pavlidis. Algorithms for Graphics and Image Processing. Computer Science Press, 1982.

[10] S. Ribaric and I. Fratric. A biometric identification system based on eigenpalm and eigenfingerfeatures. IEEE Transactions on Pattern Analysis and Machine Intelligence, 27(11):1698-1709, November 2005.

[11] R.K. Rowe, U. Uludag, M. Demirkus, S. Parthasaradhi, and A.K. Jain. A multispectral whole-hand biometric authentication system. Proc. of Biometrics Symposium Biometric Consortium Conference, pages 1-6, September 2007.

[12] W. Shu and D. Zhang. Automated personal identification by palmprint. Optical Engineering, 37(8):2359-2362, 1998.

[13] X. Wang, H. Gong, H. Zhang, B. Li, and Z. Zhuang. Palmprint identification using boosting local binary pattern. In Intl. Confrence on Pattern Recognition, pages 503506, 2006.

[14] Li Wenxin, D. Zhang, and Xu Zhuoqun. Palmprint identification by fourier transform. International Journal of Pattern Recognition and Artificial Intelligence, 16(4):417432, September 2002.

[15] X. Wu, D. Zhang, and Wang K. Fisherpalms based palmprint recognition. Pattern Recognition Letters, 24:2829-2938, 2003.

[16] P. Ying-Han, T.B.J. Andrew, N.C.L David, and F. S. Hiew. Palmprint verification with moments. Journal of Computer Graphics, Visualization and Computer Vision (WSCG), 12(1-3):325-332, Febuary 2003.

[17] D. Zhang and W. Shu. Two novel characteristics in palmprint verification:datum point invariance and line feature matching. Pattern Recognition, 32(4):691-702, April 1999.

[18] D. Zhang, A. W.K. Kong, J. You, and M. Wong. Online palmprint identification. IEEE Transactions on Pattern Analysis and Machine Intelligence, 25(9):1041-1050, September 2003.

[19] L. Zhang and D. Zhang. Characterization of palmprints by wavelet signatures via directional context modeling. Systems, Man, and Cybernetics, 34(3):1335-1347, June 2004. 